XIV Российская национальная конференция по сейсмостойкому строительству и сейсмическому районированию (с международным участием). Сборник материалов

УДК 69.04

DOI 10.37153/2687-0045-2021-14-137-138

\title{
ПЕРЕДАТОЧНЫЕ И ИМПУЛЬСНЫЕ ПЕРЕХОДНЫЕ ФУНКЦИИ ПРИ РАСЧЕТЕ СИСТЕМ С КОНЕЧНЫМ ЧИСЛОМ СТЕПЕНЕЙ СВОБОДЫ С ВЫКЛЮЧАЮЩИМИСЯ СВЯЗЯМИ
}

проф., д.т.н. Чернов Ю.Т.

Одним из наиболее эффективных методов расчета линейных систем с конечным числом степеней свободы, в том числе систем с выключающимися связями является метод основанный на передаточных (ПФ) и импульсных переходных функций (ИПФ) линейных систем и их связи. При расчете подобных систем по сравнению с традиционным методом «нормальных форм» значительно сокращаются этапы расчета и, в частности, построение форм собственных колебаний, записи уравнений относительно главных координат и обратное вычисление обобщенных функций. В этом варианте решение сразу определяется относительно главных координат.

Проиллюстрируем алгоритм расчета на примере горизонтально-вращательных колебаний твердых тел (рис. 1). При выключении связи реакция системы определяется зависимостью (рис. 2):

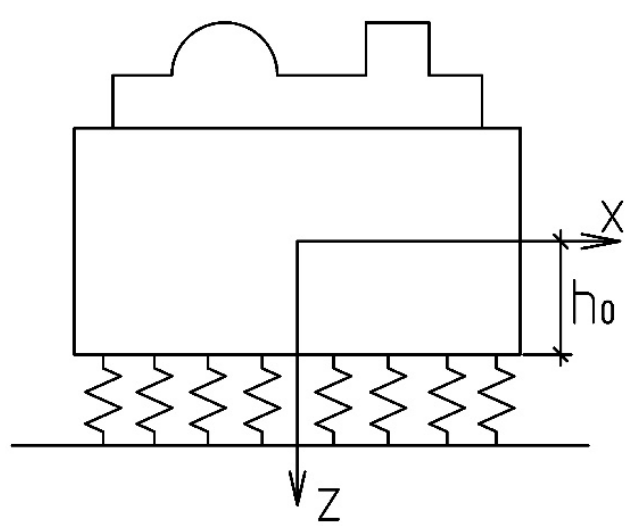

Рисунок 1

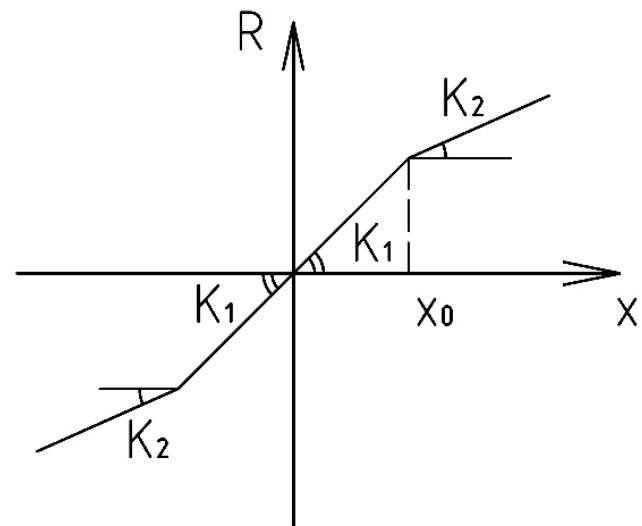

Рисунок 2

$$
R=K_{x} y \text {, при } y \leq y_{0} ; R_{\varphi}=R_{0} \mp K_{2}\left(y-y_{0}\right)
$$

Система уравнений горизонтально-вращательных колебаний в этом случае [1]:

$$
\begin{aligned}
& m_{0} \ddot{s}_{z}+K_{x} s_{z}-K_{z x} \vartheta_{y}=R h_{\omega} ; \\
& m_{0} s_{x}-K_{x z} \vartheta_{y}+K_{x} s_{x}=-m_{0} \ddot{\xi}_{0}+R
\end{aligned}
$$

где $s_{x}, \vartheta_{y}$ - горизонтальное смещение центра масс и угол поворота относительно оси у. После определения частот собственных колебаний можно определить передаточные функции: 
XIV Российская национальная конференция по сейсмостойкому строительству и сейсмическому районированию (с международным участием). Сборник материалов

$$
\begin{gathered}
H_{s}=F_{r} \sum_{r=1}^{2}(-1)^{r+1} \frac{N_{r}\left(\lambda_{i} p_{r}^{2}\right)}{p_{r}^{2}-\omega^{2}+i p_{r}^{2} \gamma_{r}} ; \\
N_{r}\left(\lambda_{i} p_{r}^{2}\right)=\frac{1}{m_{0}}\left[K_{x} \frac{K_{\vartheta}}{\rho_{o y}^{2}} K_{x z}\right] ; F_{r}=\frac{E}{p_{2}^{2}-p_{1}^{2}} ;
\end{gathered}
$$

Подобным образом определяется $H_{\theta}$, откуда следуют формулы для передаточных функций и само решение:

$$
s_{x}=F_{r} \int_{0}^{t} \ddot{\xi}_{0}(\tau) \sum_{r=1}^{2}(-1)^{r+1} U_{r}(t-\tau) d \tau \text {, при } x \leq x_{0}
$$

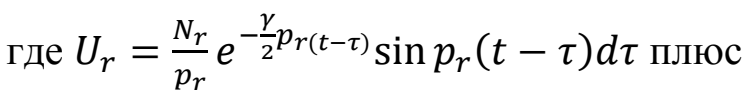

$$
s_{x \varphi}=F_{r} \int_{t_{0}}^{t} R(x, \tau) \sum_{r=1}^{2}(-1)^{r+1} U_{r} p_{r}(t-\tau) d \tau \text {, при } x>x_{0}
$$

Решение для угла поворота $\vartheta_{y}$ :

$$
\vartheta_{y}=F \int_{t_{0}}^{t} R(x, \tau) h_{0} U_{s}(t-\tau) d \tau
$$

Решения при произвольном кинематическом воздействии (5-7) вычисляются на основе шагового метода по времени (при $\Delta t<\frac{T_{r}}{20}$ ), где $T_{r}-$ период колебаний при минимальной частоте. Как следует из (2) и (3), решения, по существу, представляются в виде разложения по собственным формам колебаний.

\section{Литература}

1. Чернов Ю.Т. Вибрации строительных конструкций. Аналитические методы расчета. Основы проектирования и нормирования вибраций строительных конструкций, подвергающихся эксплуатационным динамическим воздействиям. 2-е изд., испр. и доп. М.: Изд-во АСВ, 2011. 383 с.

2. Чернов Ю.Т., Зебилила М.Д.Х. Плоские колебания массивных виброизолированных тел при произвольном смещении основания. Основания, фундаменты и механика грунтов. 2018. № 3. С. 18-22. 